\title{
MULTI-AXES MICRO GRIPPER FOR THE HANDLING AND ALIGNMENT OF FLEXIBLE MICRO PARTS
}

Development of compact and shock resistant gripper components

\author{
Christian Brecher ${ }^{1}$, Christian Peschke ${ }^{2}$, Martin Freundt ${ }^{3}$, Sven Lange ${ }^{4}$ \\ 1,2,3,4 Fraunhofer Institute for Production Technology IPT, Steinbachstrasse. 17, \\ 52072 Aachen, Germany
}

\begin{abstract}
Optical micro parts, such as glass fibres, require handling and alignment accuracies down to the sub micrometer range. Addressing this task, one aim of the Fraunhofer IPT is the development of new concepts of active gripper systems. In this context a highly integrated, adaptive, rugged and economical gripper system particularly for accurate handling and alignment of flexible micro parts down to the sub-micron level has been developed. This gripper system can be used on conventional robot systems for carrying out microassembly operations. The robot system does the pre-positioning, the tolerances necessary for the micro-assembly are subsequently realized directly at the tip of the gripper with the gripper integrated multi-axes system. Positioning systems that achieve the required positioning increments in the sub micron range are already existent. However, the problem of such systems is that they are normally highly sensitive against mechanical impact and extremely cost intensive. In this paper the development of a highly robust gripper-integrable axes system and its integration in a novel gripper design with a multi-axis adjustment system is presented.
\end{abstract}

Key words: Assembly; Flexible Micro Parts; Handling; Micro Gripper; Active Alignment; Gripper-Integrable Multi-Axes System; Gripper System.

\section{INTRODUCTION}

Assembly plays an important role in the manufacture of hybrid micro systems. In the process of micro assembly up to $80 \%$ of all arising expenses in the manufacturing process ${ }^{1}$ of a micro system are generated. Therefor flexible automated solutions are needed to reduce the costs on the one hand 
and to satisfy the continuously increasing quality demand concerning assembly accuracy in the sub-micron range $e^{2,3,4}$ on the other hand. It is the handling and assembly technology which provides the most valuable opportunities for a more variable approach, while joining techniques ${ }^{5,6}$ generally have to be selected function-specific.

Especially in the assembly of hybrid micro systems for telecommunications such as switches, star couplers, modulators, and power splitters the demands on accuracy are especially high. Even misalignments of less than $1 \mu \mathrm{m}$ between the optical components and the glass fibres can lead to significant losses in the transmission coupling of light into the fibres.

The current state of the research and technology in this field is that in many cases glass fibres are still being assembled manually. It is problematical is that accuracies in the sub-micrometer range can not be guaranteed by using manual assembly. In these cases, expensive assembly systems with active alignment strategies have to be used. On behalf of this background more research has to be conducted, particularly on the behaviour of the flexible glass fibres in robotic grippers, before automated assembly is possible. For this reason, experimental work on the optimisation of gripper design is currently being conducted at Fraunhofer IPT, to attain the highest possible repeatable positional accuracy when gripping flexible optical fibres.

In addressing this trend the Fraunhofer IPT developed an all-purpose gripper, suitable to be integrated into a conventional robot in order to reduce cost by performing micro position and alignment tasks in conventional positioning systems. Therefore the gripper is equipped with an integrated stage system allowing positioning and alignment with an accuracy of less then one micron. In order to run processes with short secondary process times the grippers design is shock resistant and compact in size by using a hydrostatic transmission. In this paper, the development of the hydrostatical transmission system and the novel gripper design with a multi-axes system will be described.

\section{DEVELOPMENT OF MINIATURISED, HIGHLY- PRECISE AND ROBUST STAGES}

For the design of the described multi-axis system compact and shock resistant stages are needed. The application requires that all components of these stages such as transmission devices and guides have to be shock resistant and compact in their dimension. In this context, the Fraunhofer IPT has developed a highly precise and robust, miniaturised stage. Based on a 


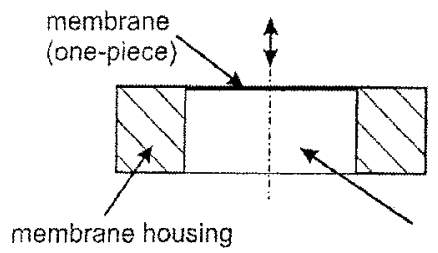

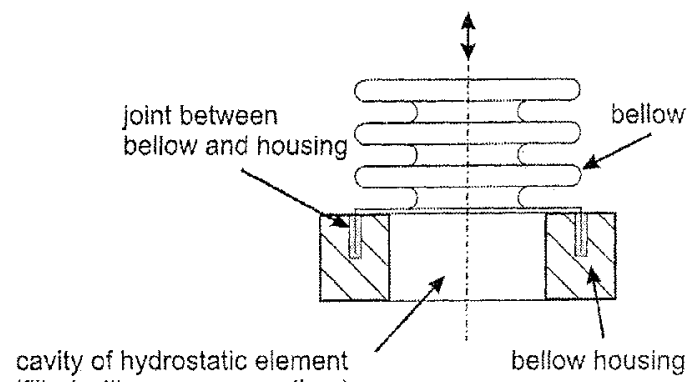

(filled with pressure medium)

Figure 1. Membrane and bellows as movable piston elements

conventional piezomotor a hydrostatic transmission principle allows extremely precise movements down to $0.1 \mu \mathrm{m}$. Currently most micro positioning systems use levers for the transmission. Comparing the principal of a lever, the hydrostatical principle is building more compact in dimension and allows for larger slip-stick free transmission of displacements, Furthermore the hydrostatical principle is more robust against high acceleration rates. This innovative principle - hydrostatic transmission - has not been used in connection with micro-positioning technology before.

The system developed at the Fraunhofer IPT consists of a reservoir filled with a hydrostatic fluid and two movable piston elements for actuation and output. As a result, these elements can be freely positioned in relation to each other, allowing an extremely compact stage design.

The development of the hydrostatic transmission element involved fundamental research into the design of the movable piston elements and the seal for these elements. Investigations on hydrostatic fluids were also necessary in order to determine, which is best dedicated for a highly precise transmission system. Solid state joints were used to allow for movement without play, hysteresis or friction. Two possible design approaches of moveable pistons as solid state elements were investigated: membrane systems and bellows (figure 1). The use of bellows allows an extremely compact design of the transmission element - compared to membranes of the same diameter, the bellows are significantly smaller and more elastic in the direction of motion. Such a system must be leak-proof in order to generate a reproducible transmission behaviour. Furthermore the element must be filled with a fluid, therefore a detachable seal is necessary. Adhesive bonds (twocomponent adhesive) were successful tested and put into use as nondetachable seals. O-sealings were tested as detachable seals but as they displaced too much volume, thereby critically increasing initial load in the 
transmission element, rectangular sealing rings were used in the final design instead.

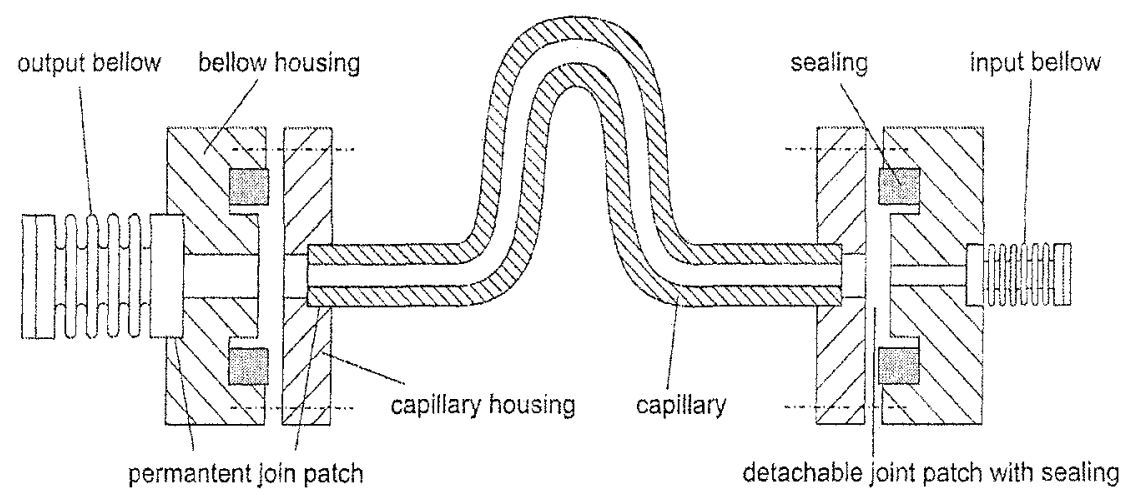

Figure 2. Design of hydrostatic transmission element for integration into gripper

Highly precise transmission through a capillary only works when friction between the fluid and the capillary is low - viscosity therefore plays an important role. Glycerine, water and pressure oils (HLP) were tested in the system. Glycerine's high viscosity (1500 times more viscous than water) makes it impossible to fill the transmission element without air bubbles. Because of their low viscosity air bubbles are not a problem when using water and HLP oils. Further tests proved that both, water and HLP oils, can generate reproducible and consistent motional behaviour. In the end, water was selected to transmit the pressure because it is easier to handle. The ratio of the effective diameters of the bellows results in a theoretical reduction ratio of 1:4.4. Figure 2 shows the design of the final hydrostatic transmission device.

As the actuator, a $20 \mathrm{~g}$ commercially available piezo-drive was chosen which uses the friction principle to move the piston rod. The minimum increments vary slightly from motor to motor between $0.6 \pm 0.1 \mu \mathrm{m}$ and $1.0 \pm 0.1 \mu \mathrm{m}$. 


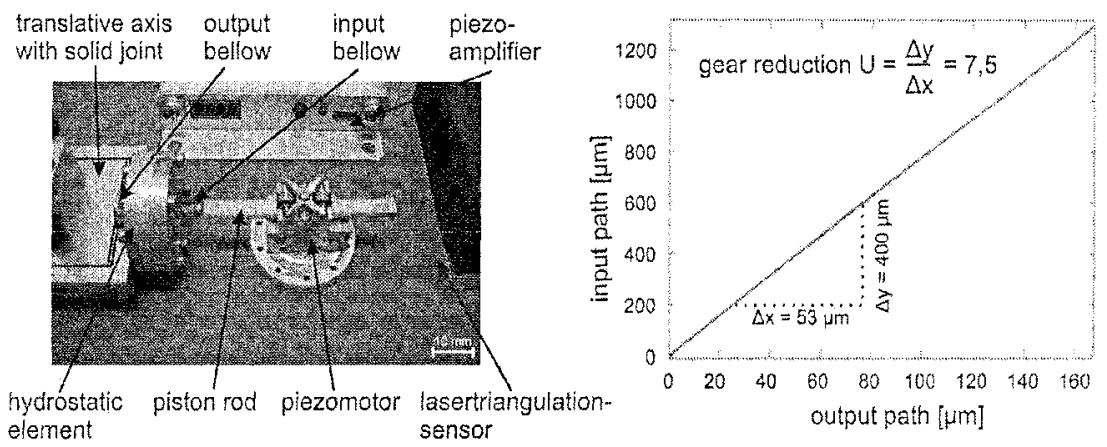

Figure 3. Prototype setup of a single-axis high precise positioning unit
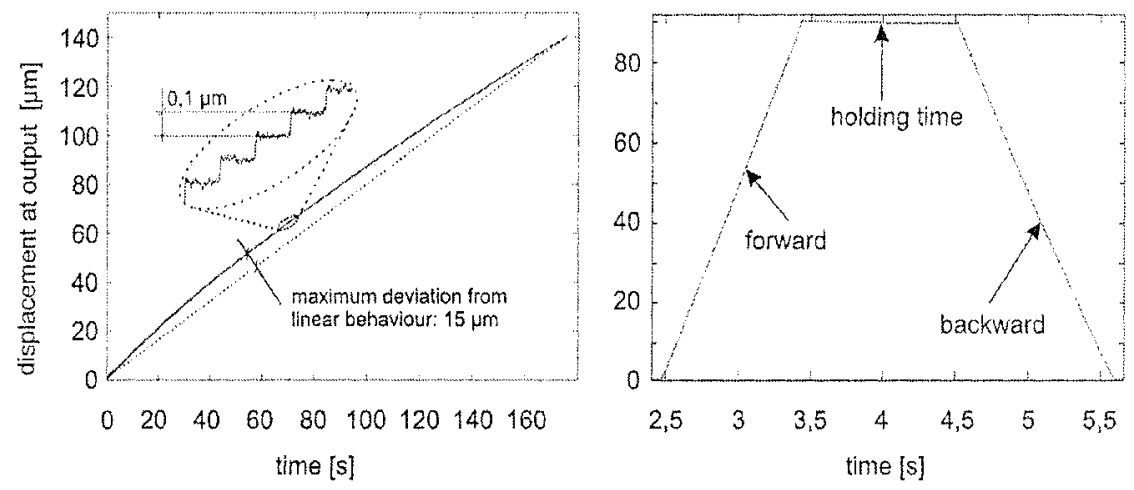

Figure 4. Motional properties of the hydrostatic transmission element

Using the described piezomotor an arithmetical reduction ratio of at least 3.5 is needed to achieve the required precision. Having moved the motor one step along, frictional losses fix the system in its position without the need of additional power. The prototype design of this unit, consisting of the piezomotor and the hydrostatic drive and output bellows is shown in figure 3 (left). The result of measurements for transition behaviour of the drive is also shown in figure 3 (right). A reduction ration of 1:7.5 \pm 0.2 was measured in the prototype single-axis system. This deviation from the theoretical value is caused by the internal elasticity of the hydrostatic element but it has a positive effect on the minimum achievable increment.

The output bellow is coupled with a stage equipped with solid state joints, resulting in minimum increments of $0.1 \pm 0.02 \mu \mathrm{m}$. This configuration can travel up to a distance of $140 \mu \mathrm{m}$ with a maximum linear deviation of $15 \mu \mathrm{m}$ along the entire path of motion. In this configuration, the 
maximum output speed is $90 \mu \mathrm{m} / \mathrm{s}$. Figure 4 shows the results of these measurements.

\section{DEVELOPMENT OF GRIPPER SYSTEM WITH AN INTEGRATED HIGHLY PRCISE MULTI- AXIS AJUSTEMENT SYSTEM}

In order to minimise losses at coupling, particularly when assembling glass fibres in front of light-emitting elements such as VCSEL laser systems, the components must be assembled with positioning deviations in the submicron range. Initial investigations have shown that strategies are needed to compensate for positioning inaccuracies during the highly precise microassembly of flexible micro parts. In order to compensate this and other factors affecting positioning accuracy, the Fraunhofer IPT's highly precise and robust stage principle was used as the basis for an innovative and adaptive gripper system capable of highly precise alignment. Therefor a robot system does the initial pre-positioning with insufficient accuracy. The necessary precision for micro-assembly is reached by the positioning and alignment function which is provided by a miniaturised stage system integrated directly in the tip of the multi-axis micro-gripper named »Flexogrip«. In the following the details of this development are described.

The kinematics of the multi-axis gripper was designed in the context of high precision glass fibre assembly. When mounting glass fibres in relation to a laser, it is essential that the fibre is aligned along the laser's optical axis with a precision in the submicron range. The kinematics of the gripper must therefor include four highly precise axes: two translational stages perpendicular to the fibre axis and two rotation stages around each of the two translational stages. The rotational movement around the fibre itself and the translational movement along to the fibre axis are less important as the demands on precision are significantly lower (a few micrometers). Serial kinematics were used in order to make the four-axis design as compact as possible. The general gripper concept is shown in figure 5. Such an 


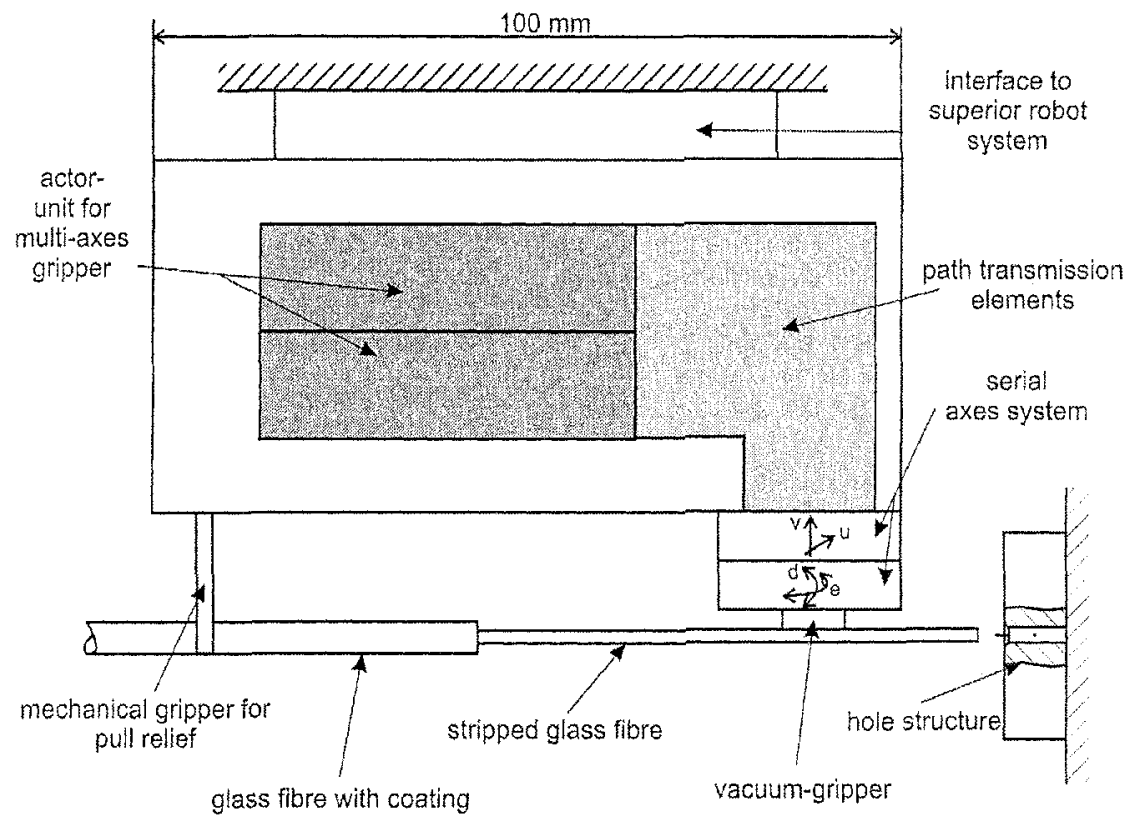

Figure 5. General concept of the multi-axis gripper

innovative gripper system, capable of moving in increments of $0.1-0.3 \mu \mathrm{m}$ by having integrated a miniaturised multi-axis system directly in the tip of the gripper, was made possible by the hydraulic transmission system described above. The minimum travel distance and angle of the gripper integrated stage system utilized to compensate for positioning errors, were determined by testing the reproducible gripping precision, the behaviour of the flexible components and positioning errors: a maximum angle of $\pm 0.2^{\circ}$ and a travel range of at least $100 \mu \mathrm{m}$ were realized.

This concept was integrated in the $\gg$ Flexogrip « which is designed to handle and precisely align flexible micro-components. The gripper weighs approx. $350 \mathrm{~g}$, is $115 \times 75 \times 40 \mathrm{~mm}^{3}$ in size and is equipped with pneumatically driven mechanical gripper used as pull relief. The movable, alignable gripper includes a high precision $\mathrm{V}$-grooved vacuum gripper to ensure that flexible micro parts are gripped accurately. Non-friction and low maintenance solid state joints act as the linear guides and bearings for rotational movements.

Figure 6 shows the final gripper without its housing. Exact measurements are still needed to determine the abilities of the combined stage system. In order to be able to monitor the process, a pressure sensor will be integrated into the vacuum-gripper and its behaviour will be characterised. The gripper 
system will also be integrated into an existing pre-positioning system at the Fraunhofer IPT in order to perform assembly trials on fibre arrays. A damping meter will be used to determine the power coupled into the assembled fibre in order to control alignment within the assembly holes of the fibre array.

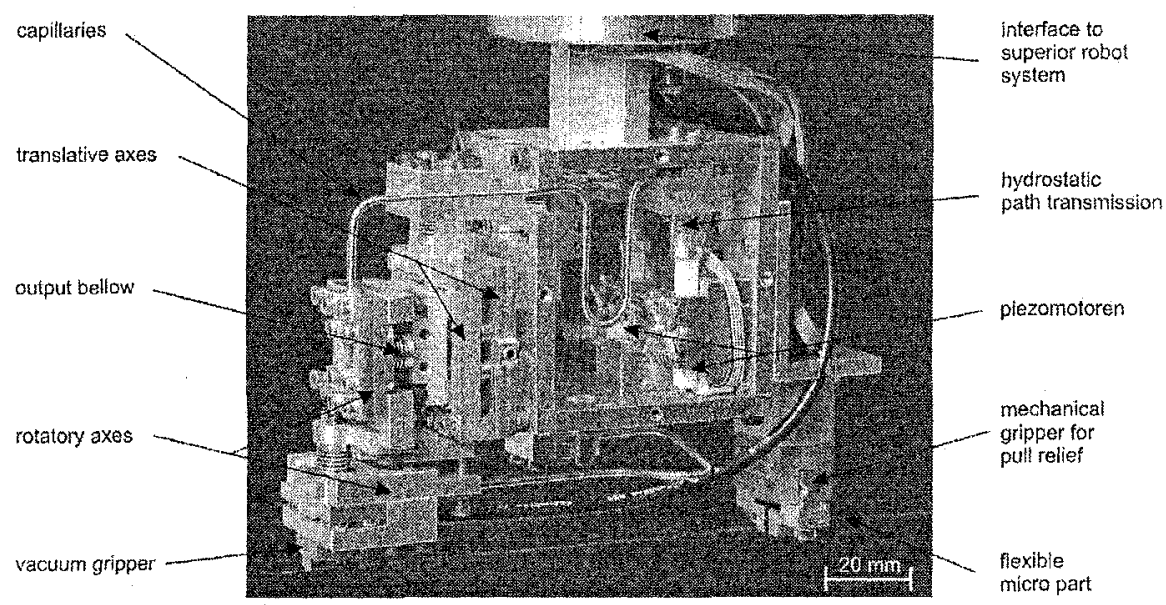

Figure 6. Multi-axis micro-gripper for handling and positioning flexible micro-components

\section{CONCLUSION AND OUTLOOK}

This paper describes the development of a unique, highly precise, robust and compact stage with a resolution of $0.1 \mu \mathrm{m}$. The stage is based on the principle of a hydrostatic transmission principle, which has not been used in micro-assembly before. The hydrostatic transmission consists of a capillary tube and metal bellows, making it very robust. The stage was accelerated up to $90 \mathrm{~m} / \mathrm{s}^{2}$ in experiments, without being damaged.

This highly precise and at the same time robust stage was put to use in the development of a gripper for the precise assembly of flexible microparts. By integrating four of these highly precise and robust stages into a gripper, it is now possible to finely align the micro-components with four degrees of freedom.

\section{ACKNOWLEDGMENT}

The authors thank the Deutsche Forschungsgemeinschaft (DFG) for the encouragement during the accomplishment of the research project (SFB 440 
"Assembly of Hybrid Micro Systems"). Furthermore the authors would like to thank the European Commission. Within the participation of the Fraunhofer IPT in the framework of the EC Network of Excellence »MultiMaterial Micro Manufacture: Technologies and Applications (4M)« parts of the results were presented to and discussed with European research and development partners.

\section{REFERENCES}

1. C. Ossmann, Back-End and Assembly Production of Cost Sensitive Microsystems, mst news 2005, Nr. 1/05, pp. 40-41

2. K. Heuer, J. Hesselbach, M. Berndt and R. Tutsch, Sensorgefuihrtes Montagesystem für die Mikromontage, Robotik 2004, VDI-Berichte Nr, 1841, ISBN 3-18-091841-1, München, 17.-18. June 2004, pp. 39-46

3. B. Petersen, Flexible Handhabungstechnik für die automatisierte Mikromontage. Dissertation, RWTH Aachen, 2003

4. G. Pokar, Untersuchung zum Einsatz von ebenen Parallelrobotern in der Mikromontage, Diss. TU Braunschweig, Vulkan, Essen, 2004

5. M. Weck and C. Peschke, Assembling hybrid microsystems - challenges and solutions, IPAS'2003, ISBN 0-85358-117-7, Q3 Digital/Litho, Loughborough, 2003

6. M. Weck and C. Peschke: Handhabung von Mikrobauteilen - Herausforderungen und Lösungen. Tagungsband: Kolloquium Mikroproduktion, ISBN 3-8027-8670-X, Vulkan, pp. 119-128, Essen, 2003 